\title{
ARTICLE High-density SNP-based genetic maps for the parents of an outcrossed and a selfed tetraploid garden rose cross, inferred from admixed progeny using the $68 \mathrm{k}$ rose SNP array
}

Mirjana Vukosavljev ${ }^{1,3}$, Paul Arens ${ }^{1}$, Roeland E Voorrips ${ }^{1}$, Wendy P C van 't Westende' ${ }^{1}$, G D Esselink ${ }^{1}$, Peter M Bourke ${ }^{1}$, Peter Cox ${ }^{2}$, W Eric van de Weg ${ }^{1}$, Richard G F Visser ${ }^{1}$, Chris Maliepaard ${ }^{1}$ and Marinus J M Smulders ${ }^{1}$

Dense genetic maps create a base for QTL analysis of important traits and future implementation of marker-assisted breeding. In tetraploid rose, the existing linkage maps include $<300$ markers to cover 28 linkage groups (4 homologous sets of 7 chromosomes). Here we used the 68k WagRhSNP Axiom single-nucleotide polymorphism (SNP) array for rose, in combination with SNP dosage calling at the tetraploid level, to genotype offspring from the garden rose cultivar 'Red New Dawn'. The offspring proved to be not from a single bi-parental cross. In rose breeding, crosses with unintended parents occur regularly. We developed a strategy to separate progeny into putative populations, even while one of the parents was unknown, using principle component analysis on pairwise genetic distances based on sets of selected SNP markers that were homozygous, and therefore uninformative for one parent. One of the inferred populations was consistent with self-fertilization of 'Red New Dawn'. Subsequently, linkage maps were generated for a bi-parental and a self-pollinated population with 'Red New Dawn' as the common maternal parent. The densest map, for the selfed parent, had 1929 SNP markers on 25 linkage groups, covering $1765.5 \mathrm{cM}$ at an average marker distance of $0.9 \mathrm{cM}$. Synteny with the strawberry (Fragaria vesca) genome was extensive. Rose ICM1 corresponded to $F$. vesca pseudochromosome 7 (Fv7), ICM4 to Fv4, ICM5 to Fv3, ICM6 to Fv2 and ICM7 to Fv5. Rose ICM2 corresponded to parts of $F$. vesca pseudochromosomes 1 and 6, whereas ICM3 is syntenic to the remainder of Fv6.

Horticulture Research (2016) 3, 16052; doi:10.1038/hortres.2016.52; Published online 26 October 2016

\section{INTRODUCTION}

Garden roses are tetraploid woody perennials from the genus Rosa (family Rosaceae, subfamily Rosidae). Roses have been cultivated since 5000 years, for ornamental purposes but also for food products (hips and petals), pharmaceuticals and perfumes. ${ }^{1-3}$ The high popularity of roses, the range of uses and intensive breeding activities have resulted in numerous cultivars. The frequent hybridization with and introgression from wild species into cultivated roses ${ }^{4-6}$ have led to a complex taxonomy. ${ }^{7,8}$ The result of these introgression events may be that some chromosomal regions may be more diverse than others among garden rose cultivars, ${ }^{9,10}$ and possibly genetically differentiated from those in cut rose.

Diploid rose species have 14 chromosomes $(2 n=2 x=14)$, whereas tetraploids have 28 chromosomes $(2 n=4 x=28)$. Depending on the similarity between particular chromosomes in a tetraploid rose cultivar, the mode of inheritance could be tetrasomic (random pairing of the four homologous chromosomes), disomic (strict preferential pairing of chromosomes, as in allopolyploids) or intermediate between these two, ${ }^{11,12}$ but this has not been quantified in rose. In the case of tetrasomic (random), pairing the chromosomes can form quadrivalents in meiosis, after which (parts of) sister chromatids may find themselves in the same gamete during the second meiotic division. This phenomenon is termed double reduction. It increases the homozygosity in gametes compared with what would be expected under random chromosome segregation. ${ }^{13}$ Double reduction is contingent on the occurrence of quadrivalents and on the occurrence of a crossover between the centromere and the observed locus; ${ }^{14}$ therefore, the frequency increases towards the distal ends of chromosomes.

A rose linkage map is useful for the study of rose genetics. A dense linkage map will enable localization and mapping of differentially expressed transcripts, ${ }^{15}$ genes and quantitative trait loci (QTLs) for important traits such as disease resistances ${ }^{16,17}$ and various ornamental traits, $1,4,18-20$ as a step towards markerassisted breeding (for example, ref. 21). In tetraploids, multiallelic markers, such as microsatellites, can amplify up to four different alleles in a single genotype, but single-nucleotide polymorphism (SNP) markers are biallelic, so we can only distinguish two alleles. Thus, a single microsatellite marker is generally more informative than a single SNP marker ${ }^{22}$ if it is scored co-dominantly. ${ }^{23,24}$ Development of highly polymorphic microsatellite markers ${ }^{25-29}$ and techniques for the determination of allele dosage of microsatellite markers ${ }^{30-32}$ have been developed, but it is still a laborious and time-consuming analysis. In contrast, thousands of SNP markers can be detected in parallel in one hybridization step, which compensates by far for the lower information content of a single SNP. In addition, scoring is largely automated as well. Recently, the 68k WagRhSNP array has been developed for roses, ${ }^{33}$ opening up the possibility to produce highdensity linkage maps in rose. ${ }^{34}$

\footnotetext{
${ }^{1}$ Wageningen UR Plant Breeding, Wageningen University \& Research, NL-6700 AJ Wageningen, The Netherlands and ${ }^{2}$ Roath BV, Eindhoven, The Netherlands Correspondence: MJM Smulders (rene.smulders@wur.nl)

${ }^{3}$ Current address: Pheno Geno Roses D.O.O. Bulevar oslobođenja 65a, 21000 Novi Sad, Serbia.

Received: 26 August 2016; Revised: 26 September 2016; Accepted: 30 September 2016
} 
Linkage maps in the genus Rosa have been produced for several diploid populations ${ }^{16,17,27,35-42}$ and a few tetraploid populations. ${ }^{12,26,42-44}$ The average distance between markers in those linkage maps was large, except in the integrated consensus map, ${ }^{40}$ where it was $0.88 \mathrm{cM}$ after combining information from five populations. For tetraploid maps the average marker distance was $2.4 \mathrm{cM}^{44}$ to $5.3 \mathrm{cM}^{43}$ The maximum distance between adjacent markers in the tetraploid maps was $16 \mathrm{cM}^{44}$ to $39 \mathrm{cM}^{42}$ It is unclear whether all homologous chromosomes and chromosomal regions were represented in the linkage maps.

Using SNP markers enables improving map coverage and density, and at the same time reduces the efforts and costs involved in producing the linkage map. Currently,TetraploidMap is the only publicly available software for mapping in autotetraploids. ${ }^{45}$ Although it can include markers that segregate as simplex $\times$ nulliplex as well as duplex $\times$ nulliplex markers, it is restricted in the number of markers and needs manual interaction and visual inspection, which limits its implementation. ${ }^{46,47} \mathrm{~A}$ new version has just been developed. ${ }^{48}$

The aim of this study was to generate the first highdensity genetic linkage map for tetraploid garden rose, using an approach that starts with constructing separate homologs. ${ }^{34}$ SNPs were genotyped using the rose WagRhSNP array and the SNP dosage was estimated by fitTetra. ${ }^{49}$ We also developed a strategy to disentangle offspring from different crosses in the absence of some of the parents, using the large amount of SNP scores and the information therein. The results enabled a detailed analysis of synteny of the rose genetic linkage maps with the woodland strawberry genome sequence.

\section{MATERIALS AND METHODS}

\section{Mapping population(s)}

A set of 177 seedlings was obtained that was intended to be an F1 mapping population from a cross between the two garden rose cultivars 'Red New Dawn' (RND, mother) and 'Morden Centennial' (MC). However, as will be shown in the Results section, the seedlings proved to have different origins; two subpopulations will be referred to as RND $\times$ RND (selfing of RND, 103 individuals) and RND $\times$ UP (RND $\times$ UP, 74 individuals). Genomic DNA was extracted from freeze-dried young leaves of RND, MC and 177 seedlings with the Qiagen DNeasy Plant Mini kit (250) (Qiagen, Venlo, The Netherlands) following the protocol used by Esselink et al. ${ }^{25}$ The seedlings were grown on their own roots in a greenhouse in Wageningen, The Netherlands.

\section{Microsatellite markers}

Microsatellite or simple sequence repeat markers were used to align the numbering of linkage groups to the Integrated Consensus rose linkage Map (ICM) map. ${ }^{40}$ Thirty-two markers (from Esselink et al., ${ }^{25}$ Hibrand Saint Oyant et al., ${ }^{27}$ Yan et $a .^{37}$ and Meng et al. ${ }^{50}$ ) were chosen to genotype the offspring (Supplementary Table ESM1). Amplification was in $10 \mu \mathrm{L}$ containing $1 \mu \mathrm{L}$ of $8 \mathrm{ng} \mathrm{\mu L}^{-1}$ DNA, $5 \mu \mathrm{L}$ multiplex kit (Qiagen) and $4 \mathrm{pmoL}$ of forward (labeled) and reverse primers, using $15 \mathrm{~min}$. denaturation at $95^{\circ} \mathrm{C}$. followed by 30 cycles of $94^{\circ} \mathrm{C}$ for $30 \mathrm{~s}$, ramp $1^{\circ} \mathrm{C} \mathrm{s}^{-1}$ to $50^{\circ} \mathrm{C}, 50^{\circ} \mathrm{C}$ for $30 \mathrm{~s}$, ramp $1{ }^{\circ} \mathrm{C} \mathrm{s}^{-1}$ to $72{ }^{\circ} \mathrm{C}, 72{ }^{\circ} \mathrm{C}$ for $120 \mathrm{~s}$ and a final extension at $72{ }^{\circ} \mathrm{C}$ for 10 min. One microliter of $100 \times$ diluted PCR product was mixed with $\mathrm{Hi}-\mathrm{Di}$ formamide containing GeneScan-500 LIZ size standard (Applied Biosystems, Foster (ity, CA) and separated on an ABI 3730. The output was analysed using Genemapper 4.0 (Applied Biosystems). The allele dosage was scored quantitatively. ${ }^{31}$ Nevertheless, we did not include the microsatellite markers in the genetic linkage maps as due to missing data points their location would be imprecise compared with that of the SNPs.

\section{SNP markers}

Generation of SNP data, dosage scoring and genotype calling, and map construction followed the steps described in Smulders et al. ${ }^{34}$ Step 1 concerned generating the SNP data; in step 2, these were inspected and filtered, genotypes were called, and their quality was checked; and in step 3 , markers were assigned to homologs, linkage maps were constructed and checked for consistency. For genotyping, we used the WagRhSNP Axiom SNP array, ${ }^{33}$ which contains 68893 SNPs probed from both directions. Hybridizations of all offspring plants and the parents were performed by Affymetrix (Santa Clara, CA, USA). In a tetraploid, a SNP with two alleles, $a$ and $b$, can be present in five different dosages: aaaa (nulliplex for b), aaab (simplex), aabb (duplex), abbb (triplex) and bbbb (tetraplex). We used fitTetra ${ }^{49}$ to score allele dosages. FitTetra uses a mixture model approach. The two probes for each SNP were fitted as independent markers. Missing scores were assigned if the dosage of a sample could not be assigned with sufficient confidence (assignment probability $<0.95$ ) or if the total signal intensity was too low.

In step 2, we initially judged for each SNP if the quality was acceptable based on: (a) the number of missing data, (b) the number of conflicting scores for replicated samples, (c) match of $\mathrm{F} 1$ progeny segregation to 1 of the 20 expected disomic or tetrasomic segregation patterns and (d) match of parental dosages with the F1 segregation. We selected only those SNPs for which both probes passed this quality check, but only if $<4 \%$ of the F1 dosages differed between the probes and the results of both probes matched the same segregation pattern. The dosage with the highest probability was selected.

The results of step $2 \mathrm{c}$, the match for possible segregation patterns, prompted us to recheck whether all seedlings were really offspring of the presumed parents, as for a large number of SNPs the segregation in the progeny did not agree with an $\mathrm{F} 1$ and/or the expectation based on the scored parental dosages. A principle component analysis (PCO) plot of the 177 seedlings using pairwise genetic distances based on all markers, made with NTSYS $2.10,{ }^{51}$ produced one cloud of samples. We then generated PCO plots of the seedlings based on pairwise genetic distances calculated only for SNPs that were monomorphic in one of the intended parents. Based on these plots, two underlying, admixed populations were manually separated. We then repeated step 2 for these two populations.

\section{Linkage map construction}

We followed Bourke et al. $^{13}$ in naming the segregation types of markers based on the dosages in both parents. For step 3, the linkage map construction, SNPs with the following segregation types are most informative: simplex $\times$ nulliplex (one allele in one parent, allele absent in the other, segregation 1:1), duplex $\times$ nulliplex (two copies of the allele in one parent, absent in the other) and simplex $\times$ simplex (one copy of the SNP allele in both parents). We first assigned simplex $\times$ nulliplex markers to linkage groups. As the estimates of recombination frequencies and log of odds (LOD) scores for simplex $\times$ nulliplex markers in coupling phase are the same for tetraploids as for diploids, ${ }^{23}$ JoinMap $4.1^{52}$ could be used for linkage group detection. The only exception is when double reduction occurs, but this is expected to occur only at a low frequency. For instance, Bourke et al. ${ }^{13}$ found frequencies $\sim 6 \%$ or more towards the distal regions of the chromosomes in autotetraploid potato. Our simplex $\times$ nulliplex SNPs were analysed using JoinMap in coupling phase for each parent separately in the cross-pollinated population and selfed population. $A X^{2}$ goodness-of-fit test was performed on the segregation data of all markers, and the markers deviating significantly $(P<0.05)$ from the expected 1:1 or 1:2:1 ratios were removed from the analysis. The SNPs were grouped into linkage groups on the basis of a logarithm of odds ratio (LOD for independence $>4$ ).

The SNPs in the linkage groups were separated into four homologous chromosomes using the assigned phase and the recombination frequency estimates, where possible. The recombination frequency between markers at the same position on different homologs is expected to be $1 / 3^{53}$ which corresponds to $\sim 39.5 \mathrm{cM}$ when using the Kosambi mapping function. If markers from different homologs are mapped together (as if they were on the same homolog), gaps of $\sim 40 \mathrm{cM}$ are therefore expected between different homologous chromosomes; such gaps were identified and used to separate the homologs. Each parental map is expected to have a total of 28 linkage groups, corresponding to the seven chromosomes and four coupling phase linkage groups per chromosome. To connect homologs within parental genomes and chromosomes across parental genomes, we subsequently added so-called bridge markers to the simplex $\times$ nulliplex segregating markers: duplex $\times$ nulliplex markers, which segregate 1:4:1 in an autotetraploid, and simplex $\times$ simplex markers, which segregate 1:2:1. Recombination frequencies and LOD scores between these markers, as well as with the simplex $\times$ nulliplex markers, in coupling phase were obtained by maximum likelihood estimation from the pairwise frequencies of the dosages of the markers in the mapping population. These estimates were assembled in so-called 'pairwise data files' and imported into JoinMap. A grouping tree was generated on the basis of a LOD threshold of 4. For linkage map construction, we used the weighted least squares regression algorithm of Joinmap with default settings. 
a

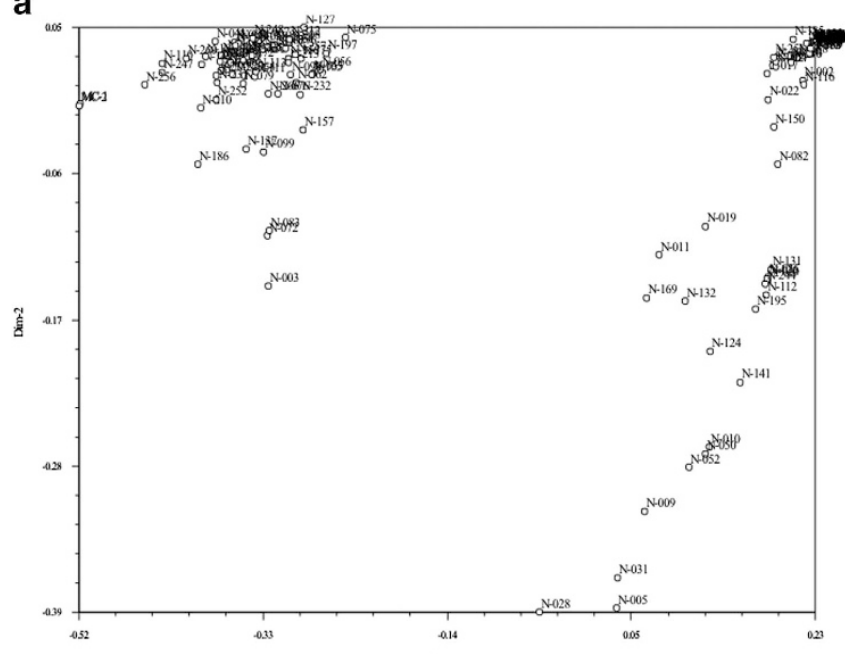

Dim-1

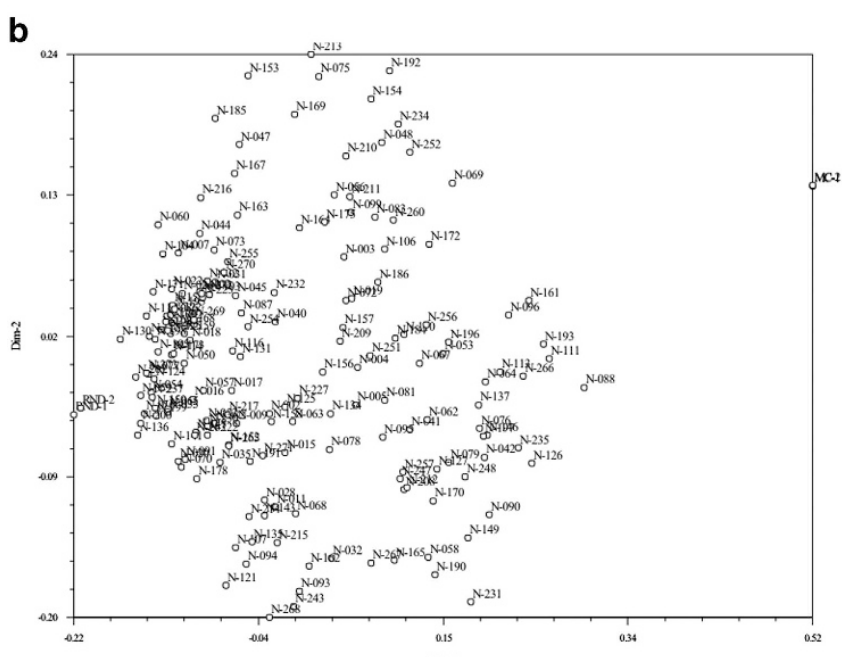

Figure 1. PCO analysis of the progeny based on pairwise genetic distances using only the SNP markers that were monomorphic (nulliplex or quadruplex) in RND (a, based on 18683 markers) and MC (b, based on 17935 markers). In (a), the first principal coordinate explained $52 \%$ and the second $29 \%$ of the variation; for (b), this was $56 \%$ and $27 \%$, respectively. The results in (a) indicate that the population consists of two subpopulations, and two pollen donors have been involved in crosses: A (the cluster to the left, 103 offspring) and $B$ (the cluster to the right, 74 offspring). The results in (b) give no indication that more than a single mother was involved.

A second method to connect homologs was by determining which of the SNPs that mapped to different homologs were derived from the same contig. For this, we used the name of the SNP on the array (Supplementary Table 2 of Koning-Boucoiran et $a .^{33}$ ), as it includes the contig number, followed by a number that indicates the position of the SNP on the contig.

Third, the homologs were also indirectly anchored by comparing the position of the contig DNA sequence from which the mapped SNP(s) were derived, with that of the most similar sequence in the Fragaria vesca genome ${ }^{54}$ version 2.0 (https://www.rosaceae.org/species/fragaria_vesca/ genome_v2.0.a1). The BLASTN was done using a sliding window with wordlength $w=9$ and $w=11$, selecting the highest hit if above a cutoff E-value of $10^{-5}$. The more stringent $w=11$ BLASTN mapped only $34 \%$ of the markers that were mapped under the more relaxed $w=9$ settings, but the synteny pattern was clearer. This BLASTN analysis also enabled visualization of the synteny between rose and $F$. vesca using Circos. ${ }^{55}$

We adopted the linkage group numbering of the ICM. ${ }^{40}$ The assignment of linkage group numbers to our linkage maps was carried out using microsatellite markers from the ICM map, but the orientation of the maps generated here is that of the $F$. vesca pseudochromsomes. Linkage maps were drawn with MapChart. ${ }^{56}$

\section{RESULTS}

A strategy to distinguish subpopulations based on selected SNP scores

In the 177 offspring plants of the intended cross RND $\times M C$, only $\sim 55 \%$ of the simplex $\times$ nulliplex SNP markers from RND fitted the expected segregation ratios, and only $17 \%$ of those from MC (not shown). Among these markers, we observed triplex and quadruplex allele dosages that were not expected, given the parental SNP dosages. Most signal intensities were within acceptable ranges, suggesting that it was not a technical issue but possibly due to the presence of outcrossed plants.

We therefore went back to the SNP selection steps and included the SNPs that had been filtered out in step $2 \mathrm{c}$ based on expected segregation ratios. Visualizing the population structure with all markers in a PCO did not produce clear groups among the seedlings. To improve the resolution, we generated two PCO plots for all offspring using pairwise similarities based on selected SNP markers, namely, those for which RND (Figure 1a) or MC (Figure 1b) were monomorphic (nulliplex or quadruplex). These markers are uninformative for one parent and can show the genetic relationships due to the other parent(s) at a higher resolution. The PCO plots indicated that, as expected, there was no differentiation based on the markers that were informative from the maternal side (Figure $1 \mathrm{~b}$ ), but the paternally informative PCO (Figure 1a) plot indicated two clusters of samples, which was interpreted as two subpopulations. This points to two different fathers. Thus, we divided the initial population into two populations: population A (consisting of 103 offspring, the left cluster in Figure 1a) and B (consisting of 74 offspring, the cluster to the right in Figure 1a). In both populations, the genotype of MC could not explain the segregation in the progeny and thus it was rejected as the pollen parent. Population $B$ was named RND $\times U P$, a cross of RND with an 'unknown parent' (UP). On the basis of genotype configurations of RND and the offspring, the marker genotype for UP could be reconstructed.

For the larger of the two subpopulations (population A), the set of segregating markers that passed all quality criteria (except concordance with the-unknown-paternal parent) consisted of 13941 markers, of which very few were simplex $\times$ nulliplex segregating markers. Not a single marker segregated duplex $x$ nulliplex, but many segregated simplex $\times$ simplex. As the inferred dosage of the parents was the same for the vast majority of the markers, we assumed that this population may be the result of selffertilization (selfing). To confirm this assumption, we looked at the single dosage markers in RND. If this population is derived from selfing, then all SNPs that segregate simplex $\times$ nulliplex from RND in the RND $\times$ UP population would have to segregate as simplex $\times$ simplex in this population. Of the 1411 simplex $\times$ nulliplex markers in RND $\times$ UP, 1099 were also scored in population A and 1061 of them (96.5\%) segregated as simplex $\times$ simplex. Of the 943 simplex $\times$ simplex markers in the RND $\times$ UP, population 689 were also scored in population $A$ and for all markers the segregation was consistent with simplex $\times$ simplex. Therefore, we concluded that population $A$ most likely originated from selfing and named it RND $\times$ RND. As an additional indication that population RND $\times$ RND arose from selfing, the progeny of RND $\times$ RND had a significantly lower heterozygosity than RND (0.55 vs $0.69 ; P=0.001)$ across all markers.

\section{Linkage map construction}

For the RND $\times$ UP population, we produced linkage maps for both parents. A total of 2513 SNPs (1411 simplex $\times$ nulliplex, 942 simplex $\times$ simplex and 160 duplex $\times$ nulliplex markers) were used for the construction of a genetic linkage map of RND, whereas for 
Table 1. Linkage map length and number of markers for the paternal RND and maternal UP maps of the RND $\times$ UP population and the map of RND from the selfed population

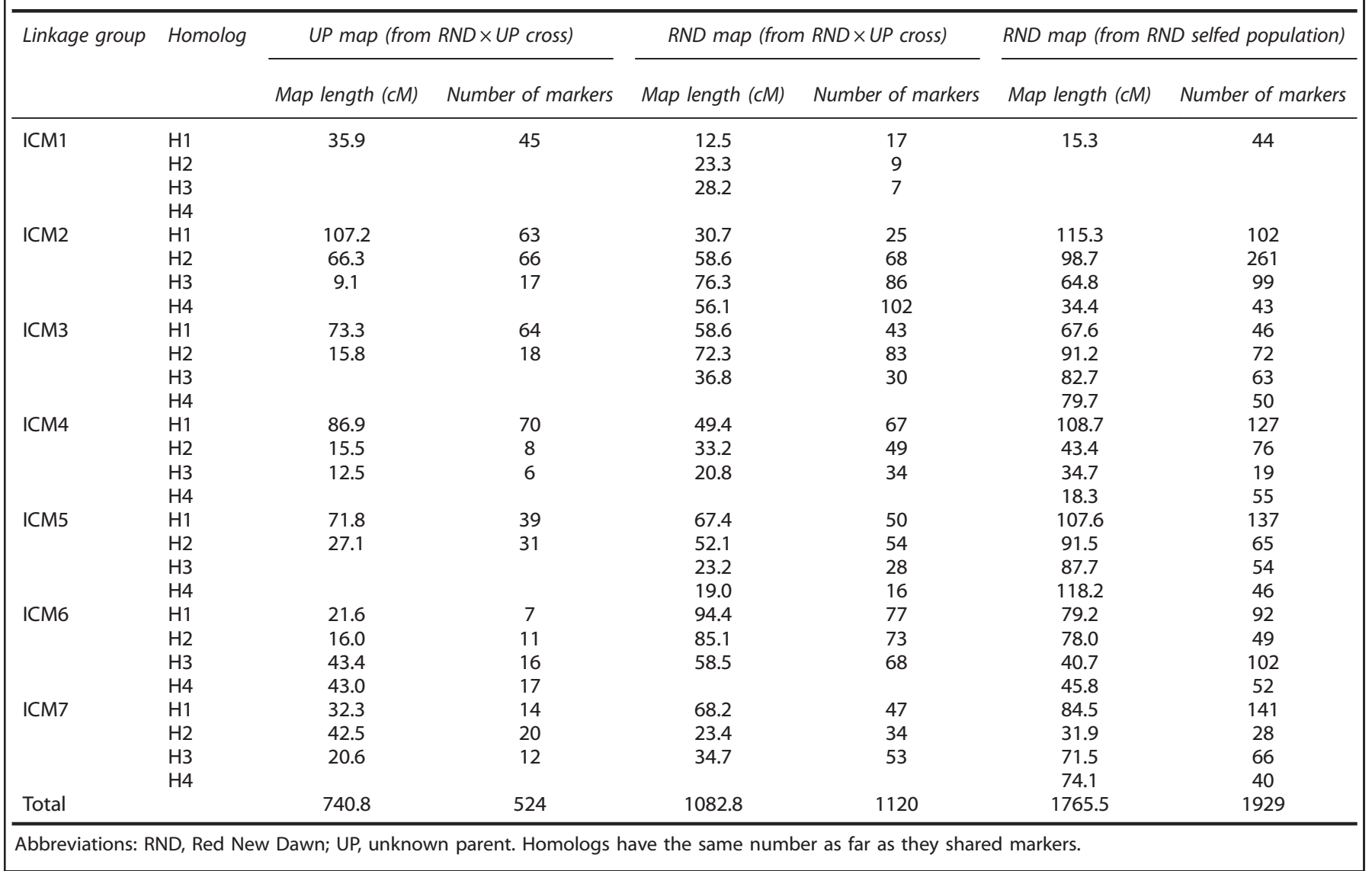

the UP map, 1760 SNPs (615 simplex $\times$ nulliplex, 942 simplex $\times$ simplex and 203 duplex $\times$ nulliplex markers) were used.

The resulting RND linkage map included 1120 SNPs assigned to 23 linkage groups with a median distance between markers of $0.96 \mathrm{cM}$ and a maximum distance between two consecutive markers of $17.5 \mathrm{cM}$. The linkage groups varied in size from 12.5 to $94.4 \mathrm{cM}$ (Table 1), adding up to a total map length of $1082.8 \mathrm{cM}$. The 23 linkage groups have been assigned to the seven chromosomes of the ICM linkage map. For two chromosomes, all four homologs were present; for five chromosomes $(1,3,4,6$ and 7), one homolog was missing.

The resulting UP linkage map included 524 SNPs (Table 1) distributed over 18 linkage groups. The length varied from 9.1 to $107.6 \mathrm{cM}$ with a mean interval distance between loci of $1.4 \mathrm{cM}$ and a maximum distance between two consecutive markers of $13.2 \mathrm{cM}$. In total, this linkage map spanned $740.8 \mathrm{cM}$ (Table 1). Only for chromosome 6, all four homologs were present; for chromosomes 2, 4, and 7, one homolog was missing; for chromosomes 3 and 5, two homologs were missing; and for chromosome 1, three homologs were missing.

Using the selfed progeny, a denser RND linkage map was produced (one homolog is shown in Figure 2). Here 1929 SNPs were mapped to 25 linkage groups, spanning $1765.5 \mathrm{cM}$ (Table 1). The length of the linkage groups varied from 15.2 to $118.2 \mathrm{cM}$. The average marker density was $0.9 \mathrm{cM}$ and the maximum distance between two consecutive markers was $25.4 \mathrm{cM}$. On the linkage map of this selfed population, most of the markers were simplex $\times$ simplex. Simplex $\times$ nulliplex markers did not occur, with the exception of six $\mathrm{S} \times \mathrm{N}$ markers that were mapped to the linkage group ICM3.

As expected, most (14\%) markers were shared between the two RND maps (Table 1). The largest number of shared markers among maps was on ICM3, which was also the linkage group with the largest number of markers in all maps (24.2-26.1\% of all mapped markers). No markers were shared for ICM1. The three parental maps have been aligned using the common markers, as far as the homologs shared markers. Only one homolog is shown here (ICM2.2 in Figure 2, see below) but all homologs can be drawn in MapChart ${ }^{56}$ using the linkage group files provided in Supplementary zipfile ESM5.

\section{Synteny with Fragaria}

For the parental RND linkage map, contig sequences of 379 SNP markers were successfully blasted to the strawberry genome, under stringent conditions (Supplementary Table ESM3), whereas 1112 markers mapped under less stringent BLAST conditions (at $w=9$ ). For the UP map, 191 and 510 markers could thus be located on the $F$. vesca genome sequence (Supplementary Table ESM4). For the RND map of the RND $\times$ RND population, 651 and 1800 markers had a hit (Supplementary Table ESM2). Under stringent conditions, a total of 1221 markers from the three maps were located on the $F$. vesca genome sequence (Table 2 ).

Most of the markers from one rose linkage group (84-98\%, except for ICM2) were located on a single $F$. vesca pseudochromosome, and in largely the same order (colinear), which indicates a high level of macro-synteny between rose and strawberry. 


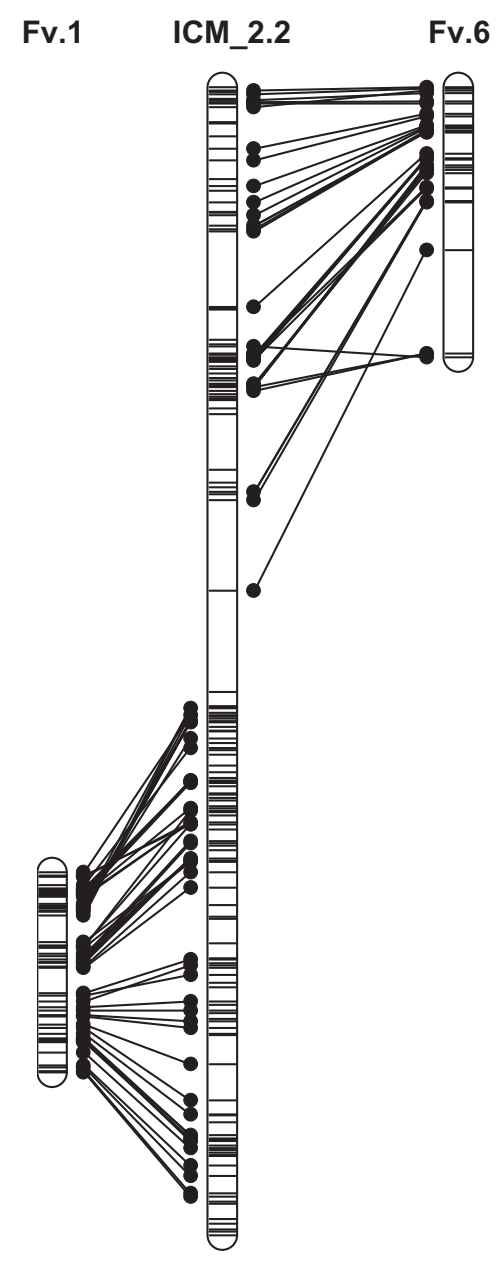

Figure 2. An example of the colinearity of the rose and strawberry genomes. Rose linkage group ICM2 homolog 2 (ICM_2.2) of the map of RND from the RND $\times$ RND population as constructed with SNP markers in the center, and the synteny with two Fragaria vesca pseudochromosomes (Fv6 and Fv1) indicated to the left and to the right. ICM_2.2 positions in CM; Fv in Mbp. Marker names and positions, and the MapChart files of the complete RND linkage map are in Supplementary zipfile ESM5, all BLASTN hits to $F$. vesca in Supplementary file ESM6. Using MapChart, ${ }^{56}$ maps of all other homologs can be visualized by opening the corresponding MapChart file.

This picture emerged both under the stringent BLAST conditions (wordlength 11, Table 2) and under less stringent conditions (wordlength 9), which gave a hit for 3422 markers, that is, almost three times as many, but with a low number of possibly accidental similaries on all other pseudochromosomes (Supplementary Tables ESM2-4).

Garden rose ICM1 corresponded to Fragaria pseudochromosome 7 (Fv7), ICM4 to Fv4, ICM5 to Fv3, ICM6 to Fv2 and ICM7 to Fv5. Rose ICM2 corresponded to parts of Fragaria pseudochromosomes 1 and 6 (Figure 2), whereas these parts were largely colinear. Rose ICM3 is the remainder of Fv6 (Table 2, Supplementary file ESM6). Generally, the Circos plot (Figure 3) is consistent with the results of Gar et al. ${ }^{44}$ and Kirov et al., ${ }^{57}$ but our dense map makes it possible to locate the breakpoint in Fv6 (Figure 2) between 18 and $20 \mathrm{Mbp}$. A small piece (from 17 to $21 \mathrm{Mbp}$ ) of Fv1 may be present at the end of ICM2. The resolution is not sufficient to detect with certainty whether other small translocations exist. The Circos plot also shows that the two genomes are largely colinear.

\begin{tabular}{|lccccccccc|}
\hline Table 2. Synteny with strawberry \\
\hline Rose linkage group (ICM) & \multicolumn{7}{c|}{ Fragaria vesca pseudochromosome } \\
\cline { 2 - 8 } & Fv1 & $F v 2$ & $F v 3$ & $F v 4$ & $F v 5$ & $F v 6$ & $F v 7$ \\
\hline 1 & 1 & 2 & 0 & 1 & 0 & 0 & $\mathbf{4 7}$ \\
2 & $\mathbf{1 6 0}$ & 0 & 1 & 2 & 10 & $\mathbf{1 5 5}$ & 4 \\
3 & 18 & 3 & 1 & 1 & 3 & $\mathbf{1 3 2}$ & 0 \\
4 & 1 & 0 & 1 & $\mathbf{1 7 3}$ & 2 & 1 & 0 \\
5 & 0 & 0 & $\mathbf{1 6 6}$ & 2 & 6 & 2 & 0 \\
6 & 1 & $\mathbf{1 9 5}$ & 3 & 0 & 0 & 0 & 0 \\
7 & 6 & 2 & 2 & 2 & $\mathbf{1 1 4}$ & 1 & 0 \\
\hline
\end{tabular}

Number of markers of the three garden rose SNP linkage maps that could be placed on the Fragaria vesca pseudochromosomes. For this, the rose sequence contigs from which the SNPs had been derived were BLASTed to the $F$. vesca genome sequence v2.0 with wordlength 11 , and the best hit was used if above the cutoff E-value of $10^{-5}$, otherwise they were considered not mapped. In bold are the groups of markers that represent the most likely syntenous linkage groups.

\section{DISCUSSION}

We developed a strategy to disentangle an admixed offspring from two crosses, even in the absence of one of the parents, using a large number of SNP dosage scores from the WagRhSNP array and the segregation information therein. We subsequently generated high-density genetic maps for two tetraploid garden rose populations, with a SNP marker density below $1 \mathrm{cM}$ between markers, which up to now has only been obtained in the ICM map $^{40}$ by integrating five different maps.

During construction of the linkage maps, we detected a large number of presumed $\mathrm{F} 1$ offspring plants that were not in agreement with the genotypes of the putative parents, beyond the small number of off-type offspring that is a common nuisance in breeding (if problems in population uniformity are reported at all). We used subsets of SNP markers for which no segregation was expected from one of the intended parents, as a strategy to detect genetic groups related to the other parent(s). With this relatively simple procedure, and with the information present in the vast number of SNP marker data available, we were able to reconstruct two subpopulations with different parentage even in the absence of genotype information of one putative parent.

One of the two populations was the result of a selfing of the mother, variety RND. This was concluded based on several lines of evidence as follows: the absence of simplex $\times$ nulliplex segregating markers, the fact that $>96 \%$ of the RND markers that segregated as simplex $\times$ nulliplex in the RND $\times$ UP population behaved as simplex $\times$ simplex in this offspring, and the $20 \%$ lower average heterozygosity compared with the mother. To date, no studies on self-compatibility in garden roses have been published, whereas breeders' experiences are confidential. Self-pollinated flowers of the diploid $R$. rugosa Thunb. wilt after pollination, suggesting the existence of gametophytic self-incompatibility, ${ }^{58}$ but Nybom et al. ${ }^{59}$ indicated that polyploid species appear to be fully self-fertile. Self-fertility has been overlooked in breeding, and our finding indicates a need to better manage pollinations while making controlled crosses in a greenhouse. On the other hand, self-fertility makes it possible to fix highly valued traits by one or two rounds of selfing. Up to now, it is unclear to what extent selfing has played a role in commercial breeding in roses.

We constructed three linkage maps for garden roses employing simplex $\times$ nulliplex, simple $\times$ simplex and duplex $\times$ nulliplex segregating SNP markers. The largest linkage map (of RND from the selfed RND population) included 1929 loci and covered $1765.5 \mathrm{cM}$ with an average marker distance of $0.9 \mathrm{cM}$. Compared with earlier tetraploid rose maps with average marker distances from $2.4 \mathrm{cM}$ (homologs integrated $^{44}$ ) to $5.3 \mathrm{cM}$ (map per homolog ${ }^{43}$ ), homolog 


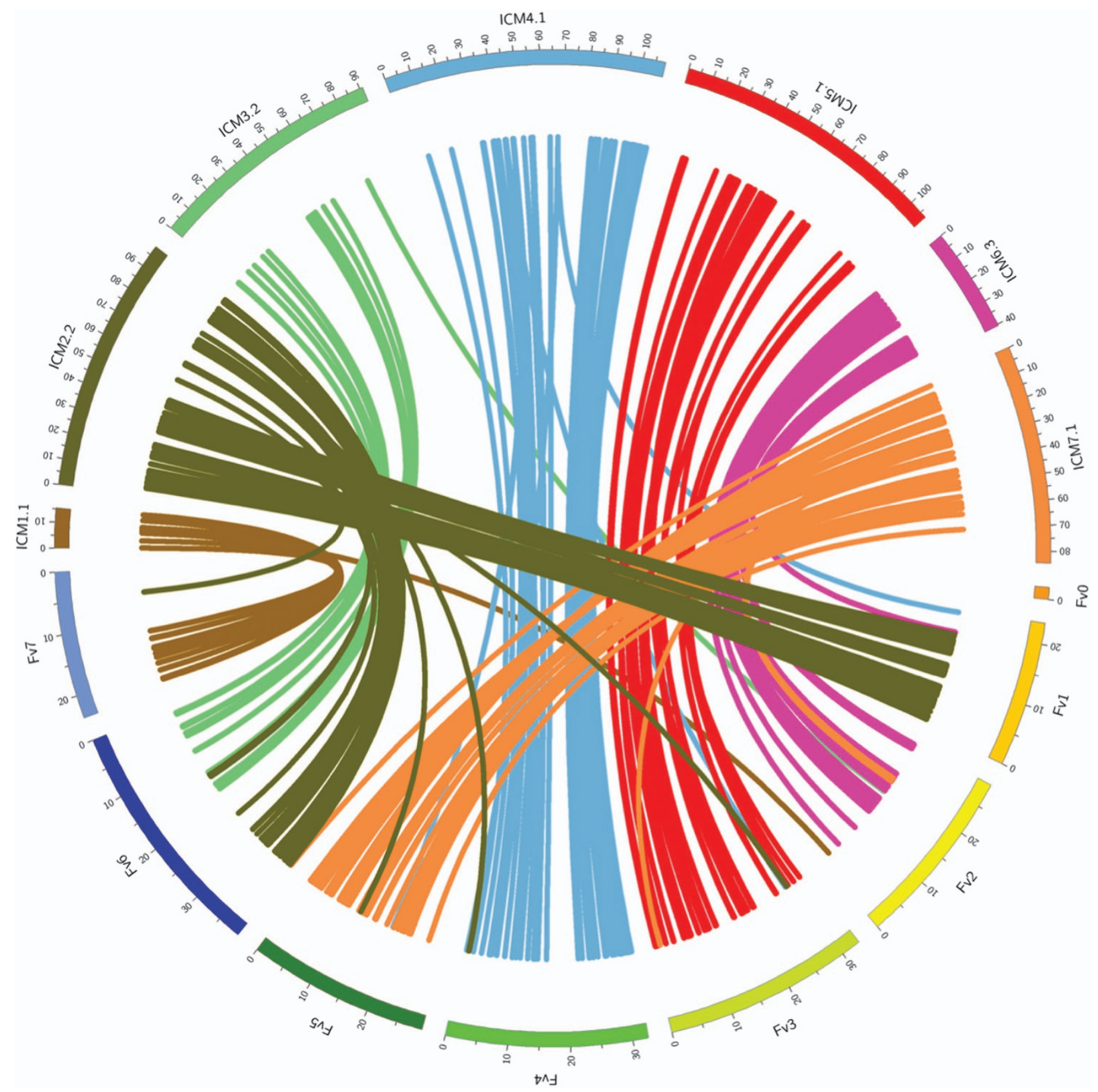

Figure 3. Circos plot of the synteny between rose and strawberry, on the basis of the contigs of the SNP markers of the RND $\times$ RND map that were BLASTed against the Fragaria vesca genome assembly (v2.0.a1), wordcount $=11$. The rose ICM homolog containing most mapped markers per linkage group are shown, with distances in CM. Fv numbering refers to $F$. vesca pseudochromosomes with distances in Mb. Marker names and positions, and the MapChart files of the complete RND linkage map are in Supplementary zipfile ESM5, all BLASTN hits to F. vesca in Supplementary file ESM6.

coverage and marker density are much improved. Some homologs are still missing, and this may not be random, as, for example, only $15 \mathrm{CM}$ of ICM1 was found in the densest RND map, whereas all other linkage groups were covered by four homologs (albeit not necessarily all complete). It may be that the level of polymorphism varies among chromosomes, possibly related to the origin of each of them in tetraploid rose, which may be composed of chromosomal segments from up to 10 species. ${ }^{3,10}$ On the other hand, it cannot be ruled out that breeding and selection (with, for example, one or two rounds of unforeseen selfing) have led to regions of (relatively high) homozygosity, as was observed in octaploid strawberry. ${ }^{60}$
Larger mapping populations will greatly increase the statistical power for QTL analyses. Software specifically being developed for polyploid maps, ${ }^{48,61}$ will enable using all markers with other segregation types, which have now remained unused, and integrating homologs. The ability to reliably generate separate haplotypes of separate homologs will contribute to our understanding of polyploid inheritance, to the estimation of heterozygosity and to determining the population structure of polyploids. $^{62}$

The genus Rosa is closely related to the genera Fragaria ${ }^{63}$ and Rubus $^{64}$ in the subfamily Rosoideae, and thus the $F$. vesca genome assembly can be used as a reference for the validation of genetic 
linkage maps in rose, ${ }^{44.65,66}$ Our synteny analysis indicated a high level of conservation between the rose and strawberry genomes. The majority of the markers that mapped on one linkage group in rose had their highest sequence similarity with the sequence of a single pseudochromosome of strawberry, with the exception of rose linkage groups 2 and 3: their markers corresponded to both strawberry pseudochromosomes 1 and 6, indicating that translocations have occurred. There is also a high degree of colinearity (as visualized in Figures 2 and 3). The macro-synteny observed in this study is in agreement with the study of Gar et al. ${ }^{44}$ in rose. Other studies between members of the Rosaceae also indicated a high level of synteny and detected strongly conserved syntenic regions among the genera Malus, Fragaria and Prunus. ${ }^{67,68}$ The high level of synteny can be used to obtain positions of nonsegregating markers or contigs and to find candidate genes both in QTL regions and around significantly associated markers in genome-wide association studies (Schultz et al., submitted). In addition, it offers an additional means of linking markers between segregating populations or genome-wide association studies, as next to multiple SNPs on one contig (up to eight SNPs on the array were derived from a single rose contig ${ }^{33}$ ) markers may also be linked between physically neighboring contigs using the Fragaria genome sequence.

The WagRhSNP array is already being used to generate a diploid rose map or $R$. chinensis 'Old Blush' $\times R$. wichurana with greatly increased marker density. ${ }^{69}$ Very dense maps of markers based on the genetic segregation data will be very helpful in the assembly of the scaffolds of the rose genome ${ }^{70}$ based on bioinformatics into correct pseudochromosomes, as was the case in the revision of the apple genome. ${ }^{71}$

\section{Conclusions}

We developed a strategy for distinguishing subpopulations that share one parent based on SNP segregation data that are monomorphic for one of the presumed parents. Using this strategy, we confirmed that selfing occurs in garden rose, which may open new possibilities for rose breeding. We used the SNP array data to produce three dense genetic linkage maps for garden roses, which in comparison with previous rose maps have significantly improved coverage of homologs and increased marker density. With these maps, we showed that synteny of rose and strawberry is extensive. The colinearity of these genomes will be very useful in finding candidate genes underlying QTLs and regions identified using genome-wide association studies.

\section{CONFLICT OF INTEREST}

The authors declare no conflict of interest.

\section{ACKNOWLEDGEMENTS}

We thank Carole Koning-Boucoiran and Tiago Miguel Santos Leonardo for advice on data analysis. This research was partly supported by the TTI Green Genetics projects 'Hyperrose' and 'Polyploids' and by the TKI Polyploids project H263 (BO-26.03-002001).

\section{AUTHOR CONTRIBUTIONS}

MJMS, PA, WEvdW, PC and CM conceived the study. MV and PC produced the crosses and provided the plant material. MV and WPCvtW extracted the DNA and prepared for hybridization. REV performed the SNP dosage scoring. MV, PA, $C M$, MJMS and REV analysed the population structure. MV, CM and PMB produced the linkage maps. GDE and PMB perfomed the synteny analyses. MV, PA, REV, RGFV and MJMS wrote the manuscript. All authors have read and approved the final text.

\section{REFERENCES}

1 Debener T, Linde M. (2009) Exploring complex ornamental genomes: the rose as a model plant. Crit Rev Plant Sci 2009; 28: 267-280.

2 Gudin S. Rose: genetics and breeding. In: Janick J (ed.). Plant Breeding Review, vol. 17. John Wiley \& sons: New York, USA, 2000; pp159-189.

3 Smulders MJM, Arens P, Koning-Boucoiran CFS, Gitonga VW, Krens F, Atanassov A et al. In: Kole C (ed.). Wild Crop Relatives: Genomics and Breeding Resources Plantation and Ornamental Crops. Springer Verlag: Berlin, Heidelberg, Germany, 2011; pp 243-275.

4 Bendahmane M, Dubois A, Raymond O, Bris ML. Genetics and genomics of flower initiation and development in roses. J Exp Bot 2013; 64: 847-857.

5 Raymond O, Just J, Dubois A, Vergne P, Szecsi J, Bendahmane M. Rose genomics: challenges and perspectives. Acta Hortic 2015; 1087: 35-40.

6 Liorzou M, Alix Pernet A, Li S, Chastellier A, Thouroude T, Michel G et al. Nineteenth century French rose (Rosa sp.) germplasm shows a shift over time from a European to an Asian genetic background. J Exp Bot 2016; 67: 4711-4725.

7 Koopman WJM, Wissemann V, De Cock K, Van Huylenbroeck J, De Riek J, Sabatino GJ et al. AFLP markers as a tool to reconstruct complex relationships: a case study in Rosa (Rosaceae). Am J Bot 2008; 95: 353-366.

8 De Riek J, De Cock K, Smulders MJM, Nybom H. AFLP-based population structure analysis as a means to validate the complex taxonomy of dogroses (Rosa section Caninae). Mol Phylogenet Evol 2013; 67: 547-559.

9 Vukosavljev M, Zhang J, Esselink GD, Van 't Westende WPC, Cox P, Visser RGF et al. Genetic diversity and differentiation in roses: a garden rose perspective. Sci Hortic 2013; 162: 320-332.

10 Zhang J, Esselink GD, Che D, Fougère-Danezan M, Arens P, Smulders MJM. The diploid origins of allopolyploid rose species studied using single nucleotide polymorphism haplotypes flanking a microsatellite repeat. J Hortic Sci Biotechnol 2013; 88: 85-92.

11 Jackson RC, Jackson JW. Gene segregation in autotetraploids: prediction from meiotic configurations. Am J Bot 1996; 83: 673-678.

12 Koning-Boucoiran CFS, Gitonga VW, Yan Z, Dolstra O, van der Linden CG, van der Schoot J et al. The mode of inheritance in tetraploid cut roses. Theor Appl Genet 2012a; 125: 591-607.

13 Bourke PM, Voorrips RE, Visser RGF, C Maliepaard C. The double reduction landscape in tetraploid potato as revealed by a high-density linkage map. Genetics 2015; 201: 853-863.

14 Ronfort J, Jenczewski E, Bataillon T, Rousset F. Analysis of population structure in autotetraploid species. Genetics 1998; 150: 921-930.

15 Dubois A, Carrere S, Raymond O, Pouvreau B, Cottret L, Roccia A et al. Transcriptome database resource and gene expression atlas for the rose. BMC Genom 2012; 13: 638.

16 Linde M, Hattendorf A, Kaufmann H, Debener T. Powdery mildew resistance in roses: QTL mapping in different environments using selective genotyping. Theor Appl Genet 2006; 113: 1081-1092.

17 Dugo ML, Satovic Z, Millán T, Cubero Jl, Rubiales D, Cabrera A et al. Genetic mapping of QTLs controlling horticultural traits in diploid roses. Theor Appl Genet 2005; 111: 511-520.

18 Kaufmann H, Qiu X, Wehmeyer J, Debener T. Isolation, molecular characterization, and mapping of four rose MLO orthologs. Front Plant Sci 2012; 3: 244.

19 Henz A, Debener T, Linde M. Identification of major stable QTLs for flower color in roses. Mol Breeding 2015; 35: 190.

20 Roman H, Rapicault M, Miclot AS, Larenaudie M, Kawamura K, Thouroude T et al. Genetic analysis of the flowering date and number of petals in rose. Tree Genet Genom 2015; 11: 85.

21 Zhang X, Zhang J, Zhang W, Yang T, Xiong Y, Che D. Transcriptome sequencing and de novo analysis of Rosa multiflora under cold stress. Acta Physiol Plant 2016; 38: 164.

22 Yang $X$, Lv Y, Pang X, Wang Z, Li X, Feng S et al. A unifying framework for bivalent linkage analysis of allotetraploids. Brief Bioinform 2012; 14: 96-108.

23 Hackett CA, McLean K, Bryan GJ. Linkage analysis and QTL mapping using SNP dosage data in a tetraploid potato mapping population. PLOS ONE 2013; 8: e63939.

24 Hackett CA, Bradshaw JE, Bryan GJ. QTL mapping in autotetraploids using SNP dosage information. Theor Appl Genet 2014; 127: 1885-1904.

25 Esselink GD, Smulders MJM, Vosman B. Identification of cut rose (Rosa hybrida) and rootstock varieties using robust sequence tagged microsatellite site markers. Theor Appl Genet 2003; 106: 277-286.

26 Zhang LH, Byrne DH, Ballard RE, Rajapakse S. Microsatellite marker development in rose and its application in tetraploid mapping. J Am Soc Hort Sci 2006; 131: 380-387.

27 Hibrand-Saint Oyant L, Crespel L, Rajapakse S, Zhang L, Foucher F. Genetic linkage maps of rose constructed with new microsatellite markers and locating QTL controlling flowering traits. Tree Genet Genom 2008; 4: 11-23.

28 Park YH, Ahn SG, Choi YM, Oh HJ, Ahn DC, Kim JG et al. Rose (Rosa hybrida L.) EST-derived microsatellite markers and their transferability to strawberry (Fragaria spp.). Sci Hortic 2010; 125: 733-739. 
29 Vukosavljev M, Esselink GD, Van 't Westende, Cox P, Visser RG, Arens $P$ et al. Efficient development of highly polymorphic microsatellite markers based on polymorphic repeats in transcriptome sequences of multiple individuals. Mol Ecol Resour 2015; 15: 17-27.

30 Esselink GD, Nybom H, Vosman B. Assignment of allelic configuration in polyploids using the MAC-PR (microsatellite DNA allele counting-peak ratios) method. Theor Appl Genet 2004; 109: 402-408.

31 Vukosavljev M, Di Guardo M, van de Weg WE, Arens P, Smulders MJM. Quantification of allele dosage in tetraploid roses. ScienceMED 2012; 3: 277-282.

32 Van Dijk T, Noordijk Y, Dubos T, Bink MCAM, Visser RGF, van de Weg WE. Microsatellite allele dose and configuration establishment (MADCE): an integrated approach for genetic studies in allopolyploids. BMC Plant Biol 2012; 12: 25.

33 Koning-Boucoiran CFS, Esselink GD, Vukosavljev $M$, van 't Westende WP, Gitonga VW, Krens FA et al. Using RNA-Seq to assemble a rose transcriptome with more than 13,000 full-length expressed genes and to develop the WagRhSNP 68k Axiom SNP array for rose (Rosa L.). Front Plant Sci 2014; 6: 249.

34 Smulders MJM, Voorrips RE, Esselink GD, Santos Leonardo TM, Van 't Westende WPC, Vukosavljev $\mathrm{M}$ et al. Development of the WagRhSNP Axiom SNP array based on sequences from tetraploid cut roses and garden roses. Acta Hortic 2015; 1064: 177-184.

35 Debener T, Mattiesch L. Construction of a genetic linkage map for roses using RAPD and AFLP markers. Theor Appl Genet 1999; 99: 891-899.

36 Crespel L, Chirollet M, Durel CE, Zhang D, Meynet J, Gudin S. Mapping of qualitative and quantitative phenotypic traits in Rosa using AFLP markers. Theor Appl Genet 2002; 105: 1207-1214.

37 Yan Z, Denneboom C, Hattendorf A, Dolstra O, Debener T, Stam P et al. Construction of an integrated map of rose with AFLP, SSR, PK, RGA, RFLP, SCAR and morphological markers. Theor Appl Genet 2005; 110: 766-777.

38 Remay A, Lalanne D, Thouroude T, Le CF, Hibrand-Saint Oyant L, Foucher F. A survey of flowering genes reveals the role of gibberellins in floral control in rose. Theor Appl Genet 2009; 119: 767-781.

39 Kawamura K, Hibrand-Saint Oyant L, Crespel L, Thouroude T, Lalanne D, Foucher F. Quantitative trait loci for flowering time and inflorescence architecture in rose. Theor Appl Genet 2011; 122: 661-675.

40 Spiller M, Linde M, Hibrand-Saint Oyant L, Tsai CJ, Byrne DH, Smulders MJ et al. Towards a unified genetic map for diploid roses. Theor Appl Genet 2011; 122: 489-500

41 Moghaddam HH, Leus L, De Riek J, Van Huylenbroeck J, Van Bockstaele E. Construction of a genetic linkage map with SSR, AFLP and morphological markers to locate QTLs controlling pathotype-specific powdery mildew resistance in diploid roses. Euphytica 2012; 184: 413-427.

42 Yu C, Luo L, Pan H, Guo X, Wan H, Zhang Q. Filling gaps with construction of a genetic linkage map in tetraploid roses. Front Plant Sci 2015; 5: 796.

43 Rajapakse S, Byrne DH, Zhang L, Anderson N, Arumuganathan K, Ballard RE. Two genetic linkage maps of tetraploid roses. Theor Appl Genet 2001; 103: 575-583.

44 Gar O, Sargent DJ, Tsai C-J, Pleban T, Shalev G, Byrne DH et al. An autotetraploid linkage map of rose (Rosa hybrida) validated using the strawberry (Fragaria vesca) genome sequence. PLOS ONE 2011; 6: e20463.

45 Hackett CA, Milne I, Bradshaw JE, Luo Z. TetraploidMap for Windows: linkage map construction and QTL mapping in autotetraploid species. J Hered 2007; 98: 727-729.

46 Voorrips RE, Maliepaard C. The simulation of meiosis in diploid and tetraploid organism using various genetic models. BMC Bioinformatics 2012; 13: 248.

47 Koning-Boucoiran CF, Smulders MJM, Krens FA, Esselink GD, Maliepaard C. SNP genotyping in tetraploid cut roses. Acta Hortic 2012; 953: 351-356.

48 Preedy KF, Hackett CA. A rapid marker ordering approach for high-density genetic linkage maps in experimental autotetraploid populations using multidimensional scaling. Theor Appl Genet 2016; doi: 10.1007/s00122-016-2761-8.

49 Voorrips RE, Gort G, Vosman B. Genotype calling in tetraploid species from bi-allelic marker data using mixture models. BMC Bioinformatics 2011; 12: 172.

50 Meng J, Li D, Yi T, Yang J, Zhao X. Development and characterization of microsatellite loci for Rosa odorata var. gigantea Rehder \& EH Wilson (Rosaceae). Conserv Genet 2009; 10: 1973-1976.

51 Rohlf FJ. NTSYS-pc: Numerical Taxonomy and Multivariate Analysis System, Version 2.1, Exeter Software: Setauket, New York, USA, 2000.

52 Van Ooijen JW. JoinMap 4, Software for the Calculation of Genetic Linkage Maps in Experimental Populations. Kyazma BV: Wageningen, Netherlands, 2006.
$53 \mathrm{Qu} \mathrm{L}$, Hancock JF. Detecting and mapping repulsion-phase linkage in polyploids with polysomic inheritance. Theor Appl Genet 2001; 103: 136-143.

54 Shulaev V, Sargent DJ, Crowhurst RN, Mockler TC, Folkerts O, Delcher AL et al. The genome of woodland strawberry (Fragaria vesca). Nat Genet 2011; 43: 109-116.

55 Krzywinski M, Schein J, Birol I, Connors J, Gascoyne R, Horsman D et al. Circos: An information aesthetic for comparative genomics. Genome Res 2009; 19: 1639-1645.

56 Voorrips RE. MapChart: software for the graphical presentation of linkage maps and QTLs. J Hered 2002; 93: 77-78.

57 Kirov I, Van Laere K, De Riek J, De Keyser E, Van Roy N, Khrustaleva L. Anchoring linkage groups of the Rosa genetic map to physical chromosomes with TyramideFISH and EST-SNP Markers. PLoS ONE 2014; 9: e95793.

58 Ueda Y, Ando TT. Pollination in Rosa rugosa Thunb. ex Murray. Acta Hort 1996; 424: 309-310.

59 Nybom H, Werlemark G, Esselink DG, Vosman B. Sexual preferences linked to rose taxonomy and cytology. Acta Hortic 2005; 690: 21-27.

60 Sargent DJ, Passey T, Šurbanovski N, Lopez Girona E, Kuchta P, Davik J et al. A microsatellite linkage map for the cultivated strawberry (Fragaria $\times$ ananassa) suggests extensive regions of homozygosity in the genome that may have resulted from breeding and selection. Theor Appl Genet 2012; 124: 1229.

61 Bourke PM, Voorrips RE, Kranenburg T, Jansen J, Visser RGF, Maliepaard C. Integrating haplotype-specific linkage maps in tetraploid species using SNP markers. Theor Appl Genet 2016; doi: 10.1007/s00122-016-2768-1.

62 Dufresne F, Stift M, Vergilino R, Mable BK. Recent progress and challenges in population genetics of polyploid organisms: an overview of current state-of-theart molecular and statistical tools. Mol Ecol 2014; 23: 40-69.

63 Potter D, Ericksson T, Evans RC et al. Phylogeny and classification of Rosaceae. Plant Syst Evol 2007; 266: 5-43.

64 Bushakra JM, Stephens MJ, Atmadjaja AN, Lewers KS, Symonds VV, Udall JA et al. Construction of black (Rubus occidentalis) and red (R. idaeus) raspberry linkage maps and their comparison to the genomes of strawberry, apple, and peach. Theor Appl Genet 2012; 125: 311.

65 Zorrilla-Fontanesi Y, Cabeza A, Torres AM et al. Development and bin mapping of strawberry genic-SSRs in diploid Fragaria and their transferability across the Rosoideae subfamily. Mol Breeding 2011; 27: 137.

66 Longhi S, Giongo L, Buti M, Surbanovski N, Viola R, Velasco R et al. Molecular genetics and genomics of the Rosoideae: state of the art and future perspectives. Hortic Res 2014; 1: 1.

67 Villanova S, Sergent D, Arus P, Monfort A. Synteny conservation between two distantly-related Rosaceae genomes: Prunus (the stone fruits) and Fragaria (the strawberry). BMC Plant Biol 2008; 6: 67.

68 Illa E, Sargent DJ, Lopez Girona E, Bushakra J, Cestaro A, Crowhurst R et al. Comparative analysis of rosaceous genomes and the reconstruction of a putative ancestral genome for the family. BMC Evol Biol 2011; 11: 9.

69 Foucher F, Hibrand-Saint Oyant L, Hamama L et al. Towards the rose genome sequence and its use in research and breeding. Acta Hortic 2015; 1064: 167-175.

70 Bendahmane M, Just J, Vergne P, Raymond O, Dubois A, Szcesi J et al. The Rose Genome Sequencing Initiative, Prospects and Perspectives. Abstract W659. Plant and Animal Genome XXIV Conference 2016. Available at https://pag.confex.com/ pag/xxiv/webprogram/Paper19873.html.

71 Bianco L, Cestaro A, Linsmith G, Muranty H, Denancé C, Théron A et al. Development and validation of the Axiom Apple480K SNP genotyping array. Plant J 2016; 86: 62-74.

This work is licensed under a Creative Commons Attribution 4.0 International License. The images or other third party material in this article are included in the article's Creative Commons license, unless indicated otherwise in the credit line; if the material is not included under the Creative Commons license, users will need to obtain permission from the license holder to reproduce the material. To view a copy of this license, visit http://creativecommons.org/licenses/ by/4.0/

(c) The Author(s) 2016

Supplementary Information for this article can be found on the Horticulture Research website (http://www.nature.com/hortres) 